\title{
Fibonacci-Lucas identities and the generalized Trudi formula
}

\author{
Taras Goy $^{1}$ and Mark Shattuck ${ }^{2}$ \\ ${ }^{1}$ Faculty of Mathematics and Computer Science \\ Vasyl Stefanyk Precarpathian National University \\ 57 Shevchenko St., 76018 Ivano-Frankivsk, Ukraine \\ e-mail: taras.goyepnu.edu.ua \\ 2 Department of Mathematics, University of Tennessee \\ 37996 Knoxville, TN, USA \\ e-mail: shattuck@math.utk.edu
}

Received: 29 February $2020 \quad$ Revised: 11 August $2020 \quad$ Accepted: 20 August 2020

\begin{abstract}
In this paper, we evaluate determinants of several families of Hessenberg matrices having Fibonacci numbers as their nonzero entries. By the generalized Trudi formula, these determinant identities may be written equivalently as formulas for the Lucas numbers in terms of the Fibonacci. We provide both algebraic and combinatorial proofs of our determinant results. The former makes use of expansion along columns and induction, while the latter draws upon the definition of the determinant as a signed sum over the symmetric group and uses parity-changing involutions.
\end{abstract}

Keywords: Hessenberg matrix, Fibonacci number, Determinant, Trudi formula, Lucas number. 2010 Mathematics Subject Classification: 05A19, 11B39, 15B05.

\section{Introduction}

Let $F_{n}$ and $L_{n}$ denote the $n$-th Fibonacci and Lucas numbers, both satisfying the recurrence $w_{n}=w_{n-1}+w_{n-2}$ for $n \geq 2$, but with the initial conditions $F_{0}=0, F_{1}=1$ and $L_{0}=2, L_{1}=1$, respectively (see, e.g., [24]).

In this paper, we find some new Fibonacci-Lucas identities which arise as determinants of certain families of Hessenberg matrices. Formulas relating determinants to Fibonacci and 
similar numbers have been an object of recent interest. In some cases, these sequences arise as determinants for certain families of matrices having integer entries, while in other cases these sequences are the actual entries of the matrix whose determinant is being evaluated. For example, Janjić [19] considered a particular type of upper Hessenberg matrix and showed its relationship with a generalization of the Fibonacci numbers (see also related work by Bicknell-Johnson and Spears [8]). Cereceda [9] provided some determinantal representations of the general terms of second and third-order linear recurrent sequences with arbitrary initial conditions, and similar work has been done by Kaygısız and Şahin in [20] for Fibonacci-type numbers in conjunction with various Hessenberg matrices. In [28], Tangboonduangjit and Thanatipanonda considered determinants of matrices whose entries are powers of the Fibonacci numbers, while Civciv [11] studied the determinant of a five-diagonal matrix with Fibonacci entries. For further examples of combinatorial determinants, we refer the reader to $[18,23]$.

In $[13,15,16]$, the authors considered determinants of some families of Toeplitz-Hessenberg matrices having the Fibonacci, Lucas or Horadam (generalized Fibonacci) numbers for the nonzero entries. These determinant formulas may also be rewritten equivalently as identities involving sum of products of Fibonacci, Lucas or Horadam numbers and multinomial coefficients. Combinatorial proofs which make use of the algebraic definition of a determinant and draw upon connections between compositions and linear tilings are provided for several of the determinant formulas in $[15,16]$.

Many previous authors have established identities featuring both the Fibonacci and Lucas numbers (the so-called Fibonacci-Lucas identities). For example, Adegoke [2] derived several binomial and ordinary summation identities involving, in particular, the Fibonacci and Lucas numbers. In [10], Čerin established nice summation formulas for Fibonacci and Lucas numbers. Frontczak [12] derived expressions for sums and alternating sums of powers, up through the fourth, of Fibonacci and Lucas numbers. In [21], Keskin and Demirtürk characterized the $2 \times 2$ matrices $X$ satisfying $X^{2}=X+I$ and obtained interesting identities concerning Fibonacci and Lucas numbers. In [27], Shattuck provided tiling proofs for some Fibonacci-Lucas relations, as requested by Benjamin and Quinn in their text [6]. Further examples of Fibonacci-Lucas identities can be found in $[1,3,17,22,24,29,30]$, among others.

The organization of this paper is as follows. In the next section, we review some basic properties of Hessenberg matrices. In the third section, we establish the determinants of several families of Hessenberg matrices having Fibonacci entries by an inductive approach. Applying the generalized Trudi formula, new identities are obtained in the fourth section which express the Lucas in terms of the Fibonacci numbers and multinomial coefficients. In the final section, we provide combinatorial proofs of several of our determinant formulas via a unified approach based on the algebraic definition of the determinant. We remark that some of the results of the third and fourth sections were announced without proofs in [14].

\section{Hessenberg matrices and determinants}

A lower Hessenberg matrix $H_{n}=\left(h_{i j}\right)$ is an $n \times n$ matrix whose entries above the superdiagonal are all zero, i.e., 


$$
H_{n}=\left[\begin{array}{cccccc}
h_{11} & h_{12} & 0 & \cdots & 0 & 0 \\
h_{21} & h_{22} & h_{23} & \cdots & 0 & 0 \\
h_{31} & h_{32} & h_{33} & \cdots & 0 & 0 \\
\vdots & \vdots & \vdots & \ddots & \vdots & \vdots \\
h_{n-1,1} & h_{n-1,2} & h_{n-1,3} & \cdots & h_{n-1, n-1} & h_{n-1, n} \\
h_{n 1} & h_{n 2} & h_{n 3} & \cdots & h_{n, n-1} & h_{n n}
\end{array}\right] .
$$

Here, we consider $n \times n$ Hessenberg matrices of the form

$$
M_{n}\left(a_{0} ; a_{1}, a_{2}, \ldots, a_{n}\right)=\left[\begin{array}{cccccc}
k_{1} a_{1} & a_{0} & 0 & \cdots & 0 & 0 \\
k_{2} a_{2} & a_{1} & a_{0} & \cdots & 0 & 0 \\
\vdots & \vdots & \vdots & \ddots & \vdots & \vdots \\
k_{n-1} a_{n-1} & a_{n-2} & a_{n-3} & \cdots & a_{1} & a_{0} \\
k_{n} a_{n} & a_{n-1} & a_{n-2} & \cdots & a_{2} & a_{1}
\end{array}\right]
$$

where $a_{0} \neq 0$ and $a_{i} \neq 0$ for at least one $i>0$. Such matrices have been studied, for example, in $[25,32]$.

Expanding the determinant of (1), which we will denote by $\operatorname{det}\left(M_{n}\right)$, repeatedly along the last column, we obtain the recurrence

$$
\operatorname{det}\left(M_{n}\right)=\left(-a_{0}\right)^{n-1} k_{n} a_{n}+\sum_{i=1}^{n-1}\left(-a_{0}\right)^{i-1} a_{i} \operatorname{det}\left(M_{n-i}\right), \quad n \geq 1 .
$$

In subsequent sections, we will investigate particular cases of the matrix (1) in which $k_{i}=i$ for all $i$ and the superdiagonal element $a_{0}$ is equal \pm 1 . To simplify our notation, we write $\operatorname{det}\left( \pm 1 ; a_{1}, a_{2}, \ldots, a_{n}\right)$ in place of $\operatorname{det}\left(M_{n}\left( \pm 1 ; a_{1}, a_{2}, \ldots, a_{n}\right)\right)$.

\section{Some Hessenberg determinants with Fibonacci entries}

We have the following explicit formulas for determinants of various Hessenberg matrices where $k_{i}=i$ for all $i$ and $a_{i}$ is derived from the Fibonacci sequence.

Theorem 3.1. For $n \geq 1$, the following formulas hold:

$$
\begin{aligned}
\operatorname{det}\left(1 ; F_{0}, F_{1}, \ldots, F_{n-1}\right) & =(-1)^{n-1}\left(L_{n}-1\right), \\
\operatorname{det}\left(-1 ; F_{0}, F_{1}, \ldots, F_{n-1}\right) & =2^{n}-L_{n}+(-1)^{n}, \\
\operatorname{det}\left(1 ; F_{1}, F_{2}, \ldots, F_{n}\right) & =(-1)^{n-1}\left(L_{n}-1\right)+1, \\
\operatorname{det}\left(1 ; F_{2}, F_{3}, \ldots, F_{n+1}\right) & =(-1)^{n-1} L_{n}, \\
\operatorname{det}\left(1 ; F_{3}, F_{4}, \ldots, F_{n+2}\right) & =(-1)^{n-1} L_{n}+1, \\
\operatorname{det}\left(-1 ; F_{3}, F_{4}, \ldots, F_{n+2}\right) & =\left(\frac{3+\sqrt{17}}{2}\right)^{n}+\left(\frac{3-\sqrt{17}}{2}\right)^{n}-L_{n}, \\
\operatorname{det}\left(1 ; F_{4}, F_{5}, \ldots, F_{n+3}\right) & =(-1)^{n-1} L_{n}+2,
\end{aligned}
$$




$$
\begin{aligned}
\operatorname{det}\left(1 ; F_{3}, F_{5}, \ldots, F_{2 n+1}\right) & =(-1)^{n-1}\left(L_{2 n}-1\right), \\
\operatorname{det}\left(1 ; F_{2}, F_{4}, \ldots, F_{2 n}\right) & =(-1)^{n-1}\left(L_{2 n}-2\right), \\
\operatorname{det}\left(1 ; F_{4}, F_{6}, \ldots, F_{2 n+2}\right) & =(-1)^{n-1} L_{2 n} .
\end{aligned}
$$

Proof. We will establish formulas (7) and (12) by induction on $n$. The other identities may be shown in a similar manner, and we omit their proofs for the sake of brevity.

Proof of (7). Clearly, the formula holds for $n=1$ and $n=2$. Suppose it is true in the $n-1$ case, where $n \geq 3$. Let $D_{n}=\operatorname{det}\left(1 ; F_{3}, F_{4}, \ldots, F_{n+2}\right)$. By recurrence (2), we then have

$$
\begin{aligned}
D_{n}= & \sum_{i=1}^{n-1}(-1)^{i-1} F_{i+2} D_{n-i}+(-1)^{n-1} n F_{n+2} \\
= & \sum_{i=1}^{n-1}(-1)^{i-1} F_{i+1} D_{n-i}+\sum_{i=1}^{n-1}(-1)^{i-1} F_{i} D_{n-i}+(-1)^{n-1} n F_{n+2} \\
= & \sum_{i=0}^{n-2}(-1)^{i} F_{i+2} D_{n-i-1}+\sum_{i=-1}^{n-3}(-1)^{i+1} F_{i+2} D_{n-i-2}+(-1)^{n-1} n F_{n+2} \\
= & F_{2} D_{n-1}-\sum_{i=1}^{n-2}(-1)^{i-1} F_{i+2} D_{n-i-1} \\
& +F_{1} D_{n-1}-F_{2} D_{n-2}+\sum_{i=1}^{n-3}(-1)^{i-1} F_{i+2} D_{n-i-2}+(-1)^{n-1} n F_{n+2} \\
= & D_{n-1}-\left(D_{n-1}-(-1)^{n-2}(n-1) F_{n+1}\right) \\
& +D_{n-1}-D_{n-2}+\left(D_{n-2}-(-1)^{n-3}(n-2) F_{n}\right)+(-1)^{n-1} n F_{n+2} \\
= & (-1)^{n}\left((n-1) F_{n+1}+(n-2) F_{n}-n F_{n+2}\right)+D_{n-1} \\
= & (-1)^{n-1}\left(F_{n+1}+2 F_{n}\right)+(-1)^{n-2} L_{n-1}+1 \\
= & (-1)^{n-1} L_{n+1}+(-1)^{n} L_{n-1}+1 \\
= & (-1)^{n-1} L_{n}+1 .
\end{aligned}
$$

Consequently, formula (7) is true in the $n$ case and thus it holds for all positive integers, by induction.

Proof of (12). When $n=1$ and $n=2$, the formula is seen to hold. Suppose (12) is true for all $k \leq n-1$, where $n \geq 3$. Let $D_{n}=\operatorname{det}\left(1 ; F_{4}, F_{6}, \ldots, F_{2 n+2}\right)$. By (2) and the well-known formula $F_{2 i+1}=\sum_{k=1}^{i} F_{2 k}+1$, we then have

$$
\begin{aligned}
D_{n} & =\sum_{i=1}^{n-1}(-1)^{i-1} F_{2 i+2} D_{n-i}+(-1)^{n-1} n F_{2 n+2} \\
& =\sum_{i=1}^{n-1}(-1)^{i-1} F_{2 i+1} D_{n-i}+\sum_{i=1}^{n-1}(-1)^{i-1} F_{2 i} D_{n-i}+(-1)^{n-1} n F_{n+2} \\
& =\sum_{i=1}^{n-1}(-1)^{i-1}\left(\sum_{k=1}^{i} F_{2 k}+1\right) D_{n-i}+\sum_{i=0}^{n-2}(-1)^{i} F_{2 i+2} D_{n-i-1}+(-1)^{n-1} n F_{2 n+2}
\end{aligned}
$$




$$
\begin{aligned}
& =\sum_{i=1}^{n-1} \sum_{k=1}^{i}(-1)^{i-1} F_{2 i-2 k+2} D_{n-i}+\sum_{i=1}^{n-1}(-1)^{i-1} D_{n-i} \\
& +F_{2} D_{n-1}-\sum_{i=1}^{n-2}(-1)^{i-1} F_{2 i+2} D_{n-i-1}+(-1)^{n-1} n F_{2 n+2} \\
& =\sum_{k=1}^{n-1} \sum_{i=0}^{n-k-1}(-1)^{i+k-1} F_{2 i+2} D_{n-k-i}+\sum_{i=1}^{n-1}(-1)^{i-1} D_{n-i} \\
& +D_{n-1}-\left(D_{n-1}-(-1)^{n-2}(n-1) F_{2 n}\right)+(-1)^{n-1} n F_{2 n+2} \\
& =\sum_{k=1}^{n-1}(-1)^{k}\left(-D_{n-k}+\sum_{i=1}^{n-k-1}(-1)^{i-1} F_{2 i+2} D_{n-k-i}\right)+\sum_{i=1}^{n-1}(-1)^{i-1} D_{n-i} \\
& +(-1)^{n}(n-1) F_{2 n}+(-1)^{n-1} n F_{2 n+2} \\
& =\sum_{k=1}^{n-1}(-1)^{k}\left(-D_{n-k}+D_{n-k}-(-1)^{n-k-1}(n-k) F_{2(n-k+1)}\right)-\sum_{i=1}^{n-1}(-1)^{i} D_{n-i} \\
& +(-1)^{n-1}\left(n F_{2 n+2}-(n-1) F_{2 n}\right) \\
& =(-1)^{n} \sum_{k=1}^{n-1}(n-k) F_{2(n-k+1)}-\sum_{i=1}^{n-1}(-1)^{i} D_{n-i}+(-1)^{n-1}\left(n F_{2 n+1}+F_{2 n}\right) \\
& =(-1)^{n} \sum_{k=1}^{n-1} k F_{2 k+2}-\sum_{i=1}^{n-1}(-1)^{i} \cdot(-1)^{n-i-1} L_{2(n-i)}+(-1)^{n-1}\left(n F_{2 n+1}+F_{2 n}\right) \\
& =(-1)^{n} \sum_{k=2}^{n}(k-1) F_{2 k}+(-1)^{n} \sum_{i=1}^{n-1} L_{2 i}+(-1)^{n-1}\left(n F_{2 n+1}+F_{2 n}\right) \text {. }
\end{aligned}
$$

Thus,

$$
D_{n}=(-1)^{n}\left(\sum_{k=1}^{n} k F_{2 k}-\sum_{k=1}^{n} F_{2 k}+\sum_{i=1}^{n-1} L_{2 i}-n F_{2 n+1}-F_{2 n}\right) .
$$

Since (see, e.g., [24])

$$
\begin{aligned}
\sum_{k=1}^{n} k F_{2 k} & =n F_{2 n+1}-F_{2 n}, \quad \sum_{k=1}^{n} F_{2 k}=F_{2 n+1}-1, \\
\sum_{k=1}^{n-1} L_{2 k} & =L_{2 n-1}-1, \quad L_{2 n+1}=F_{2 n+1}+2 F_{2 n},
\end{aligned}
$$

from (14), we have

$$
\begin{aligned}
D_{n} & =(-1)^{n}\left(n F_{2 n+1}-F_{2 n}-F_{2 n+1}+1+L_{2 n-1}-1-n F_{2 n+1}-F_{2 n}\right) \\
& =(-1)^{n-1}\left(F_{2 n+1}+2 F_{2 n}-L_{2 n-1}\right) \\
& =(-1)^{n-1}\left(L_{2 n+1}-L_{2 n-1}\right)=(-1)^{n-1} L_{2 n},
\end{aligned}
$$

which completes the induction.

Combinatorial proofs of formulas (3)-(6) and (10)-(11) are provided in the final section. 


\section{Applications by the generalized Trudi formula}

In this section, we give a multinomial analogue of Theorem 3.1 using the following result.

Theorem 4.1. Let $n$ be a positive integer. Then

$$
\operatorname{det}\left(M_{n}\right)=\sum_{\sigma_{n}=n} \frac{\left(-a_{0}\right)^{n-\left|s_{n}\right|}}{\left|s_{n}\right|}\left(\begin{array}{c}
s_{1}+\cdots+s_{n} \\
s_{1}, \ldots, s_{n}
\end{array}\right)\left(\sum_{i=1}^{n} s_{i} k_{i}\right) a_{1}^{s_{1}} a_{2}^{s_{2}} \cdots a_{n}^{s_{n}},
$$

where $\sigma_{n}=s_{1}+2 s_{2}+\cdots+n s_{n},\left|s_{n}\right|=s_{1}+s_{2}+\cdots+s_{n}$ and the summation is over all nonnegative integers $s_{i}$ satisfying $\sigma_{n}=n$.

Remark 4.2. One can find the $a_{0}=-1$ case of formula (15) in [25], though expressed in a slightly different form. Also, formula (3) from [32] is (15) written in terms of triangular matrices (for further details concerning the calculus of triangular matrices and applications, see, for example, [31] and references contained therein). Note that taking $k_{i}=1$ for all $i$ in (15) yields what is known as the Trudi formula [26, p. 214]. Thus, equation (15) may be viewed as a generalized Trudi formula. If, in the matrix (1), one takes $k_{i}=i$ for all $i$, then (15) becomes

$$
\operatorname{det}\left(M_{n}\right)=n \cdot \sum_{\sigma_{n}=n} \frac{\left(-a_{0}\right)^{n-\left|s_{n}\right|}}{\left|s_{n}\right|}\left(\begin{array}{c}
s_{1}+\cdots+s_{n} \\
s_{1}, \ldots, s_{n}
\end{array}\right) a_{1}^{s_{1}} a_{2}^{s_{2}} \cdots a_{n}^{s_{n}} .
$$

This formula may also be found in [26, p. 228].

Formula (16), taken together with Theorem 3.1 above, yields the following new identities expressing the Lucas numbers in terms of the Fibonacci numbers and multinomial coefficients.

Corollary 4.2.1. For $n \geq 1$, the following formulas hold:

$$
\begin{aligned}
& L_{n}=1-n \sum_{\sigma_{n}=n} \frac{(-1)^{\left|s_{n}\right|}}{\left|s_{n}\right|} m_{n}(s) F_{0}^{s_{1}} F_{1}^{s_{2}} \cdots F_{n-1}^{s_{n}}, \\
& L_{n}=2^{n}+(-1)^{n}-n \sum_{\sigma_{n}=n} \frac{m_{n}(s)}{\left|s_{n}\right|} F_{0}^{s_{1}} F_{1}^{s_{2}} \cdots F_{n-1}^{s_{n}} \text {, } \\
& L_{n}=1+(-1)^{n}-n \sum_{\sigma_{n}=n} \frac{(-1)^{\left|s_{n}\right|}}{\left|s_{n}\right|} m_{n}(s) F_{1}^{s_{1}} F_{2}^{s_{2}} \cdots F_{n}^{s_{n}} \text {, } \\
& L_{n}=-n \sum_{\sigma_{n}=n} \frac{(-1)^{\left|s_{n}\right|}}{\left|s_{n}\right|} m_{n}(s) F_{2}^{s_{1}} F_{3}^{s_{2}} \cdots F_{n+1}^{s_{n}} \text {, } \\
& L_{n}=(-1)^{n}-n \sum_{\sigma_{n}=n} \frac{(-1)^{\left|s_{n}\right|}}{\left|s_{n}\right|} m_{n}(s) F_{3}^{s_{1}} F_{4}^{s_{2}} \cdots F_{n+2}^{s_{n}} \text {, } \\
& L_{n}=\left(\frac{3+\sqrt{17}}{2}\right)^{n}+\left(\frac{3-\sqrt{17}}{2}\right)^{n}-n \sum_{\sigma_{n}=n} \frac{m_{n}(s)}{\left|s_{n}\right|} F_{3}^{s_{1}} F_{4}^{s_{2}} \cdots F_{n+2}^{s_{n}} \text {, } \\
& L_{n}=(-1)^{n} 2-n \sum_{\sigma_{n}=n} \frac{(-1)^{\left|s_{n}\right|}}{\left|s_{n}\right|} m_{n}(s) F_{4}^{s_{1}} F_{5}^{s_{2}} \cdots F_{n+3}^{s_{n}} \text {, } \\
& L_{2 n}=1-n \sum_{\sigma_{n}=n} \frac{(-1)^{\left|s_{n}\right|}}{\left|s_{n}\right|} m_{n}(s) F_{3}^{s_{1}} F_{5}^{s_{2}} \cdots F_{2 n+1}^{s_{n}} \text {, }
\end{aligned}
$$




$$
\begin{aligned}
& L_{2 n}=2-n \sum_{\sigma_{n}=n} \frac{(-1)^{\left|s_{n}\right|}}{\left|s_{n}\right|} m_{n}(s) F_{2}^{s_{1}} F_{4}^{s_{2}} \cdots F_{2 n}^{s_{n}}, \\
& L_{2 n}=-n \sum_{\sigma_{n}=n} \frac{(-1)^{\left|s_{n}\right|}}{\left|s_{n}\right|} m_{n}(s) F_{4}^{s_{1}} F_{6}^{s_{2}} \cdots F_{2 n+2}^{s_{n}},
\end{aligned}
$$

where $\sigma_{n}=s_{1}+2 s_{2}+\cdots+n s_{n},\left|s_{n}\right|=s_{1}+\cdots+s_{n}, m_{n}(s)=\frac{\left(s_{1}+\cdots+s_{n}\right) !}{s_{1} ! \cdots s_{n} !}$ and the summation is over all nonnegative integers $s_{i}$ satisfying $\sigma_{n}=n$.

\section{Combinatorial proofs}

In this section, we provide combinatorial proofs for several of the formulas in Theorem 3.1 above making use of the determinant definition

$$
\operatorname{det}(A)=\sum_{\sigma \in \mathcal{S}_{n}}(-1)^{\operatorname{sgn}(\sigma)} a_{1, \sigma(1)} a_{2, \sigma(2)} \cdots a_{n, \sigma(n)}
$$

where $\operatorname{sgn}(\sigma)$ denotes the sign of the permutation $\sigma$. For combinatorial proofs of determinants of matrices whose entries are derived from various counting sequences, see, e.g., [4, 5, 7]. Observe that for a Hessenberg matrix $A$, the only permutations $\sigma$ that can make a nonzero contribution to the expansion of $\operatorname{det}(A)$ in (17) are those in which every cycle of $\sigma$ comprises an interval of positive integers in increasing order (where it is understood that the smallest element is first in each cycle).

Arranging cycles in increasing order of smallest elements, such $\sigma$ may be regarded as compositions of $n$, upon identifying the various cycle lengths as parts. Suppose that the Hessenberg matrix $M_{n}$ defined by (1) above has $a_{0}=1$ and $k_{i}=i$ for all $i$. Then each part of size $i$ within the aforementioned compositions, other than one possibly in the initial position, receives weight $a_{i}$, with an initial part of size $i$ receiving weight $i a_{i}$. The weight of a composition is then defined as the product of the weights of its parts, with the sign given by $(-1)^{n-m}$ where $m$ denotes the number of parts. Thus, one may view the sum in (17) in this case as a (signed) weighted sum over the set of all compositions of $n$, where the sign and weight are as defined.

Recall that $F_{n+1}$ gives the number of (linear) square-and-domino tilings of length $n$, where squares and dominos are $1 \times 1$ and $1 \times 2$ pieces, respectively, and are considered indistinguishable. Benjamin and Quinn [6] used this enumerative interpretation of $F_{n}$ in providing combinatorial interpretations for many of the identities from Vajda [29]. Let $\mathcal{F}_{n}$ denote the set of square-anddomino tilings of length $n$ if $n \geq 1$, with $\mathcal{F}_{0}$ consisting of the empty tiling. Upon labeling the positions overlaid by a member of $\mathcal{F}_{n}$, one may view tilings as coverings of the numbers $1, \ldots, n$, where a square (denoted by $s$ ) covers a single number and a domino (denoted $d$ ) covers two consecutive numbers. We will say that a square (respectively, domino) within a member of $\mathcal{F}_{n}$ corresponds to a position $i$, where $1 \leq i \leq n$, if the square (respectively, right half of the domino) covers the number $i$.

Let $a$ be a fixed positive integer. Let $\mathcal{Q}_{n}$ be the set of tilings of length $a$ in which tiles corresponding to positions am for $1 \leq m \leq n$ may be marked and whose final tile is always marked. Further, if the leftmost marked tile corresponds to position aj for some 
$j \in[n]=\{1,2, \ldots, n\}$, then one of the positions ai where $i \in[j]$ is designated. We will refer to members of $\mathcal{Q}_{n}$ as configurations. Note that only one position within a configuration is to be designated, despite there being possibly more than one marked tile. Given configuration $\lambda$, one may write $\lambda=\lambda_{1} \cdots \lambda_{r}$, where each $\lambda_{i}$ ends in a marked tile and contains no other marked tiles and some position within $\lambda_{1}$ is designated as described. We may require further, if desired, that the marked pieces ending the $\lambda_{i}$ always be of one tile type or the other. Also, one may require that the piece ending the subtiling $\lambda_{1}$ be $s, d$ or either (independently of the other $\lambda_{i}$ ), with the particular choice taken a function of the length of $\lambda_{1}$. Note in this case that the first column of the corresponding matrix would be given more generally by $1 F_{a+i_{1}}, 2 F_{2 a+i_{2}}, \ldots, n F_{n a+i_{n}}$, where $i_{1}, i_{2}, \ldots, i_{n} \in\{0,1,-1\}$.

Given $1 \leq i \leq n$, let $\mathcal{Q}_{n, i}$ denote the subset of $\mathcal{Q}_{n}$ whose members contain exactly $i$ marked tiles. Define the sign of $\lambda \in \mathcal{Q}_{n, i}$ by $(-1)^{n-i}$. If, for example, any subtiling $\lambda_{i}$ may end in either a square or a domino, then $\operatorname{det}\left(1 ; F_{a+1}, F_{2 a+1}, \ldots, F_{n a+1}\right)$ is seen to give the sum of the signs of all members of $\mathcal{Q}_{n}=\cup_{i=1}^{n} \mathcal{Q}_{n, i}$, by the definition of the determinant above. If only squares may be marked, then $\operatorname{det}\left(1 ; F_{a}, F_{2 a}, \ldots, F_{n a}\right)$ is seen to give this sum of signs. If only dominos may be marked, then $\operatorname{det}\left(1 ; F_{a-1}, F_{2 a-1}, \ldots, F_{n a-1}\right)$ gives the sum. In any case, we will refer to the determinant of order $n$ which arises as the sum of the signs of all members of $\mathcal{Q}_{n}$, and depends upon the option taken concerning the final piece of the various subtilings, as being the determinant associated with $\mathcal{Q}_{n}$.

Determinants associated with some set $\mathcal{Q}_{n}$ of configurations may be evaluated combinatorially as follows. Let $\mathcal{Q}_{n}^{*}=A_{n} \cup B_{n}$, where $A_{n}=\mathcal{Q}_{n, 1}$ and $B_{n}$ denotes the subset of $\mathcal{Q}_{n, 2}$ in which there is no tile that corresponds to a position am for some $m \geq 1$ occurring strictly between the two marked tiles. Let $a_{n}=\left|A_{n}\right|$ and $b_{n}=\left|B_{n}\right|$ for $n \geq 1$. Note that $b_{1}=0$ for all possible sets of configurations since no member of $B_{1}$ can contain two marked tiles. Given $\lambda \in \mathcal{Q}_{n}-\mathcal{Q}_{n}^{*}$, identify the first piece $p$ lying between the leftmost and the second leftmost marked tiles that corresponds to a position $a m$ for some $m \geq 1$. Let $\lambda^{\prime}$ be obtained from $\lambda$ by either marking or removing the marking from the piece $p$, whichever is applicable. One may verify that the mapping $\lambda \mapsto \lambda^{\prime}$ defines a sign-changing involution of $\mathcal{Q}_{n}-\mathcal{Q}_{n}^{*}$. Thus, the determinant associated with $\mathcal{Q}_{n}$ is given as the sum of signs of members of $\mathcal{Q}_{n}^{*}$; that is, it equals

$$
(-1)^{n-1}\left|A_{n}\right|+(-1)^{n-2}\left|B_{n}\right|=(-1)^{n-1}\left(a_{n}-b_{n}\right) .
$$

Thus, if a Hessenberg matrix $A$ is such that $\operatorname{det}(A)$ is the associated determinant for a set $\mathcal{Q}_{n}$, then the problem of evaluating $\operatorname{det}(A)$ is equivalent to determining the counting sequences $a_{n}$ and $b_{n}$ defined above. We now apply this strategy in evaluating formulas (6), (3), (5), (10) and (11) above, where $a=1$ is taken in the framework above to prove the first three formulas and $a=2$ for the last two.

\subsection{Combinatorial proof of formula (6)}

In this case, let $a=1$ with all subtilings $\lambda_{i}$ above allowed to either end in a square or a domino. Then we have $\operatorname{det}\left(1 ; F_{2}, F_{3}, \ldots, F_{n+1}\right)=(-1)^{n-1}\left(a_{n}-b_{n}\right)$, with $a_{n}=n F_{n+1}$ since members of $A_{n}$ in this case correspond to $n$-tilings in which one of the positions is designated. Considering 
whether or not the last piece within a member of $B_{n}$ is a square, we have

$$
b_{n}=(n-1) F_{n}+(n-2) F_{n-1}=(n-1) F_{n+1}-F_{n-1}
$$

and thus

$$
a_{n}-b_{n}=n F_{n+1}-\left((n-1) F_{n+1}-F_{n-1}\right)=F_{n+1}+F_{n-1}=L_{n},
$$

as desired.

\subsection{Combinatorial proof of formula (3)}

Let $\mathcal{Q}_{n}$ here correspond to the case when $a=1$ with all marked tiles being dominos. Then since $a_{n}=n F_{n-1}$ in this case, to complete the proof of (3), we must show

$$
b_{n}=(n-2) F_{n-1}-F_{n}+1, \quad n \geq 1,
$$

where we may assume $n \geq 3$. To establish (18), first consider the set $\mathcal{U}_{n}$ of all $n$-tilings ending in a domino and containing at least two dominos such that one of the first $n-2$ positions is marked. Note that $\left|\mathcal{U}_{n}\right|=(n-2)\left(F_{n-1}-1\right)$. Let $\mathcal{U}_{n}^{\prime} \subseteq \mathcal{U}_{n}$ consist of those tilings in which the marked position corresponds to some square lying between the two rightmost dominos. Note that $b_{n}=\left|\mathcal{U}_{n}\right|-\left|\mathcal{U}_{n}^{\prime}\right|$, by the definitions.

Furthermore, members of $\mathcal{U}_{n}^{\prime}$ are synonymous with ordered pairs $\rho=(\lambda, j)$, where $\lambda=\lambda^{\prime} d s^{i} d \in \mathcal{F}_{n}, i \geq 1$ and $j \in[i]$. Let $g(\rho)=s^{i-j} d \lambda^{\prime} d s^{j-1}$. Then $g$ is seen to be a bijection with the set of $(n-1)$-tilings that contain at least two dominos, of which there are $F_{n}-(n-1)$, by subtraction. Thus $\left|\mathcal{U}_{n}^{\prime}\right|=F_{n}-(n-1)$, which implies (18), as desired.

\subsection{Combinatorial proof of formula (5)}

Let $\mathcal{Q}_{n}$ in this case denote the set of configurations corresponding to $a=1$ in which marked tiles are always squares. Then clearly $a_{n}=n F_{n}$ since members of $A_{n}$ in this case correspond to $n$-tilings ending in a square in which one of the positions is marked. To establish (5), we need to show

$$
b_{n}=(n-1) F_{n}-2 F_{n-1}+1+(-1)^{n}, \quad n \geq 1,
$$

for then

$$
\begin{aligned}
\operatorname{det}\left(1 ; F_{1}, F_{2}, \ldots, F_{n}\right) & =(-1)^{n-1}\left(a_{n}-b_{n}\right)=(-1)^{n-1}\left(F_{n}+2 F_{n-1}-1+(-1)^{n-1}\right) \\
& =(-1)^{n-1}\left(L_{n}-1\right)+1 .
\end{aligned}
$$

For (19), we consider cases based on the parity of $n$. If $n$ is even, then we must show $b_{n}=(n-1) F_{n}-2\left(F_{n-1}-1\right)$. We may assume $n \geq 4$, since the $n=2$ case is clear. To determine $b_{n}$, we count equivalently $n$-tilings ending in a square in which a non-terminal position is marked, but not one covered by either half of some domino lying between the two rightmost squares. To enumerate such $n$-tilings, we subtract from $(n-1) F_{n}$ twice $k_{n}$, where $k_{n}$ counts the set of "marked" $n$-tilings ending in a square wherein there is at least one domino occurring 
between the rightmost two squares, with one of these dominos bearing a mark. Note that tilings counted by $k_{n}$ are synonymous with ordered pairs $\rho=(\lambda, j)$, where $\lambda=\lambda^{\prime} s d^{i} s$ for some $i \geq 1$ is a member of $\mathcal{F}_{n}$ and $j \in[i]$. Let $f(\rho)=d^{i-j} s \lambda^{\prime} s d^{j-1}$. Then $f$ is seen to be a bijection with the set $\mathcal{F}_{n-2}-\left\{d^{(n-2) / 2}\right\}$, whence $k_{n}=F_{n-1}-1$, as desired.

Now assume $n \geq 3$ is odd, and we need to show $b_{n}=(n-1) F_{n}-2 F_{n-1}$. In this case, first consider the set of marked $n$-tilings that end in a square, excluding $s^{n}$, wherein a non-terminal position is marked (of which there are $(n-1)\left(F_{n}-1\right)$ possibilities). From this, we subtract those tilings wherein the mark corresponds to a position covered by some domino occurring between the rightmost two squares. Applying the bijection $f$ from above, it is seen that half the cardinality of this subtracted set of tilings equals the number of $(n-2)$-tilings that contain more than one square, which is given by $F_{n-1}-\frac{n-1}{2}$. Thus, we get

$$
b_{n}=(n-1)\left(F_{n}-1\right)-2\left(F_{n-1}-(n-1) / 2\right)=(n-1) F_{n}-2 F_{n-1},
$$

as desired, which completes the proof.

\subsection{Combinatorial proof of formula (10)}

In this case, we let $a=2$ in the definition of $\mathcal{Q}_{n}$ above and allow for marked tiles (which correspond to even-numbered positions) to either be squares or dominos. Then we have

$$
\operatorname{det}\left(1 ; F_{3}, F_{5}, \ldots, F_{2 n+1}\right)=(-1)^{n-1}\left(a_{n}-b_{n}\right) \text {, }
$$

with $a_{n}=n F_{2 n+1}$ since members of $A_{n}$ correspond to $(2 n)$-tilings wherein some even position is marked. To determine $b_{n}$, first let $A_{n}^{\prime} \subseteq A_{n}$ consist of those tilings in which the mark occurs to the right of the greatest position $2 j, 0 \leq j<n$, such that $2 j+1$ is either covered by a square or the left half of a domino. From the definitions, members of $B_{n}$ are synonymous with members of $A_{n}-A_{n}^{\prime}$, whence $b_{n}=a_{n}-\left|A_{n}^{\prime}\right|$. Thus, to prove (10), it suffices to show $\left|A_{n}^{\prime}\right|=L_{2 n}-1$.

To do so, first note that members of $A_{n}^{\prime}$ may be viewed as having one of the following three forms:

(i) a tiling $\lambda=\lambda^{\prime} d$, where $\lambda^{\prime} \in \mathcal{F}_{2 n-2}$,

(ii) a tiling $\lambda=\lambda^{\prime} s s$, where $\lambda^{\prime} \in \mathcal{F}_{2 n-2}$, or

(iii) an ordered pair $(\lambda, j)$, where $\lambda=\lambda^{\prime} s d^{i} s \in \mathcal{F}_{2 n}, i \geq 1$ and $j \in[i] \cup\{0\}$.

Let $\mathcal{L}_{m}$ denote the set of tilings of length $m$ in which an initial domino may be marked (denoted by $d^{*}$ ). Then $\left|\mathcal{L}_{m}\right|=L_{m}$ for $m \geq 1$, so to complete the proof, it suffices to define a bijection from $A_{n}^{\prime}$ to $\mathcal{L}_{2 n}-\left\{d^{n}\right\}$. To do so, if $\lambda \in A_{n}^{\prime}$ is of the form (i) or (ii) above, then let $h(\lambda)=d^{*} \lambda^{\prime}$ or $s \lambda^{\prime} s$, respectively. For members of $A_{n}^{\prime}$ of form (iii), let $h((\lambda, j))=d^{i-j} s \lambda^{\prime} s d^{j}$. Note that case (iii) of $h$ misses members of $\mathcal{L}_{2 n}$ beginning and ending with $s$ since $j$ and $i-j$ cannot simultaneously be zero as $i \geq 1$, by assumption, which implies $h$ is one-to-one. Furthermore, the element $d^{n} \in \mathcal{L}_{2 n}$ has no pre-image in $A_{n}^{\prime}$ under $h$. Thus, it is seen that $h$ yields the desired bijection, which completes the proof. 


\subsection{Combinatorial proof of formula (11)}

In this case, let $a=2$ in the definition of $\mathcal{Q}_{n}$ above with the marked tiles always being squares. Then clearly $a_{n}=n F_{2 n}$, so to establish (11), we must show

$$
b_{n}=(n-1) F_{2 n}-2 F_{2 n-1}+2, \quad n \geq 1,
$$

where $n \geq 3$ may be assumed. Let $\mathcal{V}_{n}$ denote the set of marked $(2 n)$-tilings ending in a square and containing at least three squares altogether wherein some even-numbered position, not the last, is marked. Note that, by subtraction, $\left|\mathcal{V}_{n}\right|=(n-1)\left(F_{2 n}-n\right)$. Let $\mathcal{V}_{n}^{\prime} \subseteq \mathcal{V}_{n}$ consist of those tilings in which the marked position occurs strictly to the right of the third rightmost square. By the definitions, members of $B_{n}$ are synonymous with tilings in $\mathcal{V}_{n}-\mathcal{V}_{n}^{\prime}$, whence $b_{n}=\left|\mathcal{V}_{n}\right|-\left|\mathcal{V}_{n}^{\prime}\right|$. Thus, to complete the proof of (20), we must show $\left|\mathcal{V}_{n}^{\prime}\right|=2 F_{2 n-1}-n(n-1)-2$.

Members of $\mathcal{V}_{n}^{\prime}$ may be regarded as ordered pairs $\rho=(\lambda, \ell)$, where $\lambda=\lambda^{\prime} s d^{j} s d^{i-j} s \in \mathcal{F}_{2 n}$, $i \geq 1, j \in[i] \cup\{0\}$ and $\ell \in([i] \cup\{0\})-\{j\}$. Note that $\ell$ here represents the choice of position to be marked out of the possible non-terminal even positions occurring to the right of the third rightmost $s$ in $\lambda$. Let $\mathcal{F}_{2 n-2}^{\prime} \subseteq \mathcal{F}_{2 n-2}$ consist of those tilings containing at least four squares altogether. Since there are $\left(\begin{array}{l}n \\ 2\end{array}\right)+1$ members of $\mathcal{F}_{2 n-2}$ containing less than four (i.e., two or zero) squares, we have $\left|\mathcal{F}_{2 n-2}^{\prime}\right|=F_{2 n-1}-\left(\begin{array}{l}n \\ 2\end{array}\right)-1$. Now define $h: \mathcal{V}_{n}^{\prime} \rightarrow \mathcal{F}_{2 n-2}^{\prime}$ as follows. Given $\rho \in \mathcal{V}_{n}^{\prime}$ as described above, let

$$
h(\rho)= \begin{cases}\lambda^{\prime} s d^{\ell} s d^{j-\ell-1} s d^{i-j}, & \text { if } \ell<j \\ \lambda^{\prime} s d^{j} s d^{\ell-j-1} s d^{i-\ell}, & \text { if } \ell>j .\end{cases}
$$

One may verify that $h$ is a 2 -to- 1 correspondence from $\mathcal{V}_{n}^{\prime}$ onto $\mathcal{F}_{2 n-2}^{\prime}$, upon interchanging the roles of $\ell$ and $j$. Thus, we have

$$
\left|\mathcal{V}_{n}^{\prime}\right|=2\left|\mathcal{F}_{2 n-2}^{\prime}\right|=2 F_{2 n-1}-n(n-1)-2,
$$

as desired.

If instead $a_{0}=-1$ in the matrix $M_{n}$ given by (1) above, then the product derived from the superdiagonal entries within each term in the expansion (17) is precisely the sign of the corresponding permutation $\sigma$ for all $\sigma$. Thus, $\operatorname{det}(A)$ in this case gives a positive weighted sum over the set of compositions of $n$. In the case of (4), where $a_{i}=F_{i-1}$ for all $i$, to account for each term in the expansion, one may overlay the corresponding composition with a square-and-domino tiling wherein the endpoints of the various parts are covered by the right halves of dominos, with such dominos then being circled. Note that since $a_{1}=F_{0}=0$, each part must be of size at least two and hence can accommodate a domino. Furthermore, we designate one of the positions to the left of the first circled domino to account for the extra weight coming from the first part. We now provide an enumeration of the set of configurations that result.

\subsection{Combinatorial proof of formula (4)}

We may assume $n \geq 4$ in our proof since the cases of (4) when $1 \leq n \leq 3$ are easily verified. Let $\mathcal{K}_{n}$ denote the set of tilings of length $n$ in which dominos may be circled and ending in a 
circled domino, where some position to the left of or including the position corresponding to the leftmost circled domino is marked. Then $\operatorname{det}\left(-1 ; F_{0}, F_{1}, \ldots, F_{n-1}\right)$ is seen to give $\left|\mathcal{K}_{n}\right|$. Consider transforming members of $\mathcal{K}_{n}$ to binary sequences by replacing each $s$ by $1, d$ by 01 and circled $d$ by 00 , and marking the digit to the left of (possibly part of) the first occurrence of 00 which corresponds to the marked position within the original member of $\mathcal{K}_{n}$. By a slight abuse of notation, the resulting set of (marked) binary words will again be denoted by $\mathcal{K}_{n}$. We now consider the structure of these words as follows. By a unit within a binary word, we mean a subsequence of consecutive 0's and 1's ending in 00 such that there are no two adjacent 0 's occurring prior to the terminal 00 . Then members $\lambda$ of $\mathcal{K}_{n}$, written as binary words, have the property that they may be decomposed into one or more units, i.e., each $\lambda$ is expressible as $\lambda=u_{1} \cdots u_{r}$ for some $r \geq 1$ where the $u_{i}$ are units and one of the digits in $u_{1}$ is marked.

Consider the following six cases regarding how the digit in $u_{1}$ is marked:

(i) Final 0 is marked,

(ii) Marked digit is a 1 ,

(iii) Marked digit is a 0 , which is followed by a 1 ,

(iv) Next-to-last 0 is marked, with $u_{1}$ starting with 1 ,

(v) Next-to-last 0 is marked, with $u_{1}$ starting with 01 ,

(vi) Next-to-last 0 is marked, with $u_{1}=00$.

We first enumerate cases (i)-(iv) above. To aid in doing so, let $\mathcal{J}_{n}$ denote the set of binary words of length $n$ excluding those $w$ meeting one of the following:

(I) $w$ has no occurrences of 00 and ends in 1 ,

(II) $w$ has no occurrences of 00 , ends in 0 and starts with 1 , or

(III) $w$ may be decomposed as a sequence of one or more units, followed by a single 0 .

By subtraction, we have

$$
\left|\mathcal{J}_{n}\right|=2^{n}-F_{n+1}-F_{n-1}-t_{n-1}=2^{n}-L_{n}-t_{n-1}
$$

where $t_{n}$ denotes the number of binary sequences of length $n$ that may be decomposed into one or more units.

Let $\mathcal{K}_{n}^{*}$ denote the subset of $\mathcal{K}_{n}$ corresponding to cases (i)-(iv) above. We define $\phi: \mathcal{K}_{n}^{*} \rightarrow \mathcal{J}_{n}$ as follows. Let $\lambda=u_{1} \cdots u_{r} \in \mathcal{J}_{n}$. If (i) holds, then let $\phi(\lambda)=u_{2} \cdots u_{r} u_{1}$. For the next two cases, consider decomposing the unit $u_{1}$ using the position of its marked digit. If (ii) holds, then $u_{1}=\alpha \beta$, where $\alpha$ ends in 1 and contains no 00 and $\beta$ is itself a unit. In this case, let $\phi(\lambda)=u_{2} \cdots u_{r} \beta \alpha$. If (iii) holds, then we may write $u_{1}=\alpha 01 \beta$, where $\alpha$ is possibly empty such that $\alpha 0$ contains no 00 and $\beta$ is a unit. In this case, let $\phi(\lambda)=u_{2} \cdots u_{r} \beta 1 \alpha 0$. Finally, if (iv) holds, then we have $u_{1}=\alpha 00$, where $\alpha$ starts with 1 and is such that $\alpha 0$ contains no 00 , in which case we let $\phi(\lambda)=u_{2} \cdots u_{r} 0 \alpha 0$. Note that in cases (i)-(iii) above, the binary word $\phi(\lambda)$ is assured of containing at least one unit, whereas in (iv), it may or may not contain a unit depending 
on whether or not $r \geq 2$. Combining the various cases, one may verify that the mapping $\phi$ is a bijection and hence $\left|\mathcal{K}_{n}^{*}\right|=2^{n}-L_{n}-t_{n-1}$.

To complete the proof of (4), it remains to show that the cardinality of the set $\mathcal{K}_{n}-\mathcal{K}_{n}^{*}$ (whose members are those accounted for by (v) and (vi) above) is given by $t_{n-1}+(-1)^{n}$. Since clearly $\left|\mathcal{K}_{n}-\mathcal{K}_{n}^{*}\right|=2 t_{n-2}$, by the definitions, we must show $t_{n-1}+(-1)^{n}=2 t_{n-2}$. That is, we must establish the recurrence

$$
t_{n}=2 t_{n-1}+(-1)^{n}, \quad n \geq 3 .
$$

One may assume $n \geq 5$, since clearly $t_{2}=t_{3}=1$ and $t_{4}=3$. Let $\mathcal{T}_{n}$ denote the set of binary words that can be decomposed into one or more units and $\mathcal{T}_{n}^{\prime} \subseteq \mathcal{T}_{n}$ those in which the final unit $u$ starts with 0 (i.e., $u=00$ or $u=01 v$ for a unit $v$ ). Upon placing a 1 at the beginning of the final unit within a member of $\mathcal{T}_{n-1}$, to establish (21), it suffices to show $\left|\mathcal{T}_{n}^{\prime}\right|=t_{n-1}+(-1)^{n}$.

To do so, we will define a near bijection $\rho$ between $\mathcal{T}_{n-1}$ and $\mathcal{T}_{n}^{\prime}$. Let $\lambda=u_{1} \cdots u_{r} \in \mathcal{T}_{n-1}$, where the $u_{i}$ are units. We consider cases based on the final unit $u_{r}$. If $u_{r}$ starts with 1 , then let $\rho(\lambda)=u_{1} \cdots u_{r-1} 0 u_{r}$. If $u_{r}=00$, whence $r \geq 2$, then let $\rho(\lambda)=u_{1} \cdots u_{r-2} 1 u_{r-1} 00$. If $u_{r}=(01)^{j} 00$ for some $j \geq 1$, then let $\rho(\lambda)=u_{1} \cdots u_{r-2} 1 u_{r-1}(00)^{j+1}$ if $r \geq 2$ and be undefined if $r=1$ (in which case $n$ would be odd and $j=\frac{n-3}{2}$ ).

Finally, if $u_{r}=(01)^{j} 1 \alpha$, where $\alpha$ is a unit and $j \geq 1$, then let $\rho(\lambda)=u_{1} \cdots u_{r-1} 01 \alpha(00)^{j}$. If $n$ is odd, then one may verify that the mapping $\rho: \mathcal{T}_{n-1}-\left\{(01)^{(n-3) / 2} 00\right\} \rightarrow \mathcal{T}_{n}^{\prime}$ is a bijection, whence $t_{n-1}-1=\left|\mathcal{T}_{n}^{\prime}\right|$ in this case. If $n$ is even, then $\rho$ is a bijection between $\mathcal{T}_{n-1}$ and $\mathcal{T}_{n}^{\prime}-\left\{(00)^{n / 2}\right\}$ as $\lambda=(00)^{n / 2}$ would have no pre-image under $\rho$, whence $t_{n-1}=\left|\mathcal{T}_{n}^{\prime}\right|-1$ in this case. This completes the proof of (21), as desired.

\section{References}

[1] Abd-Elhameed, W. M., \& Zeyada, N. A. (2018). New identities involving generalized Fibonacci and generalized Lucas numbers, Indian J. Pure Appl. Math., 49 (3), 527-537.

[2] Adegoke, K. (2018). A new Fibonacci identity and its associated summation identities, preprint, Available online at: https: / / arxiv.org/abs/1809.06850.

[3] Azarian, M. K. (2012). Identities involving Lucas or Fibonacci and Lucas numbers as binomial sums, Int. J. Contemp. Math. Sci., 7 (45), 2221-2227.

[4] Benjamin, A. T., \& Cameron, N. T. (2005). Counting on determinants, Amer. Math. Monthly, $112(6), 481-492$.

[5] Benjamin, A. T., Cameron, N. T., \& Quinn, J. J. (2007). Fibonacci determinants - a combinatorial approach, Fibonacci Quart., 45 (1), 39-55.

[6] Benjamin, A. T., \& Quinn, J. J. (2003). Proofs that Really Count: The Art of Combinatorial Proof, Mathematical Association of America, Washington DC. 
[7] Benjamin, A. T., \& Shattuck, M. A. (2007). Recounting determinants for a class of Hessenberg matrices, Integers, 7, \#A55.

[8] Bicknell-Johnson, M., \& Spears, C. P. (1996). Classes of identities for the generalized Fibonacci numbers $G_{n}=G_{n-1}+G_{n-c}$ from matrices with constant valued determinants, Fibonacci Quart., 34 (2), 121-128.

[9] Cereceda, J. L. (2014). Determinantal representations for generalized Fibonacci and Tribonacci numbers, Int. J. Contemp. Math. Sci., 9 (6), 269-285.

[10] Čerin, Z. (2013). On factors of sums of consecutive Fibonacci and Lucas numbers, Ann. Math. Inform., 41, 19-25.

[11] Civciv, H. (2008). A note on the determinant of five-diagonal matrices with Fibonacci numbers, Int. J. Contemp. Math. Sci., 3 (9), 419-424.

[12] Frontczak, R. (2018). Sums of powers of Fibonacci and Lucas numbers: A new bottom-up approach, Notes Number Theory Discrete Math., 24 (2), 94-103.

[13] Goy, T. (2018). On some fibinomial identities, Chebyshevskii Sb., 19 (2), 56-66 (in Russian).

[14] Goy, T. (2019). On generalized Brioshci's formula and its applications, Proceedings of the 5th Conference of the Mathematical Society of the Republic of Moldova, September 28 October 1, 2019, Chisinau, Republic of Moldova, 70-73.

[15] Goy, T., \& Shattuck, M. (2019). Determinants of Toeplitz-Hessenberg matrices with generalized Fibonacci entries, Notes Number Theory Discrete Math., 25 (4), 83-95.

[16] Goy, T., \& Shattuck, M. (2019). Fibonacci and Lucas identities using Toeplitz-Hessenberg matrices, Appl. Appl. Math., 14 (2), 699-715.

[17] Hisert, G. A. (2012). A different method for deriving Fibonacci power identities, JP J. Algebra Number Theory Appl., 24 (1), 1-26.

[18] İpek, A., \& Arı, K. (2014). On Hessenberg and pentadiagonal determinants related with Fibonacci and Fibonacci-like numbers, Appl. Math. Comput., 229, 433-439.

[19] Janjić, M. (2010). Hessenberg matrices and integer sequences, J. Integer Seq., 13, Article 10.7.8.

[20] Kaygısız, K., \& Şahin, A. (2012). Determinant and permanent of Hessenberg matrix and Fibonacci type numbers, Gen. Math. Notes, 7 (2), 32-41.

[21] Keskin, R., \& Demirtürk, B. (2010). Some new Fibonacci and Lucas identities by matrix methods, Internat. J. Math. Ed. Sci. Tech., 414 (3), 379-387.

[22] Kılıç, E., Akkus, I., Ömür, N., \& Ulutaş, Y. T. (2015). Formulas for binomial sums including powers of Fibonacci and Lucas numbers, Politehn. Univ. Bucharest Sci. Bull. Ser. A Appl. Math. Phys., 77 (4), 69-78. 
[23] Kılıç, E., \& Arıkan, T. (2017). Evaluation of Hessenberg determinants via generating function approach, Filomat, 31 (15), 4945-4962.

[24] Koshy, T. (2017). Fibonacci and Lucas Numbers and Applications, Wiley, New York.

[25] Li, H., \& MacHenry, T. (2013). Permanents and determinants, weighted isobaric polynomials, and integer sequences, J. Integer Seq., 16, Article 13.3.5.

[26] Muir, T. (1960). The Theory of Determinants in the Historical Order of Development, Vol. 3, Dover Publications, New York.

[27] Shattuck, M. (2008). Tiling proofs of some Fibonacci-Lucas relations, Integers, 8, \#A18.

[28] Tangboonduangjit, A., \& Thanatipanonda, T. (2016). Determinants containing powers of generalized Fibonacci numbers, J. Integer Seq., 19, Article 16.7.1.

[29] Vajda, S. (1989). Fibonacci \& Lucas Numbers, and the Golden Section: Theory and Applications, John Wiley \& Sons, New York.

[30] Wituła, R., Słota, D., \& Hetmaniok, E. (2013). Bridges between different known integer sequences, Ann. Math. Inform., 41, 255-263.

[31] Zatorsky, R., \& Goy, T. (2016). Permanents of triangular matrices and some general theorems on number sequences, J. Integer Seq., 19, Article 16.2.2.

[32] Zatorsky, R., \& Stefluk, S. (2013). On one class of partition polynomials, Algebra Discrete Math., 16 (1), 127-133. 